\title{
A 158 MS/S JPEG 2000 CODEC WITH A BIT-PLANE AND PASS PARALLEL EMBEDDED BLOCK CODER
}

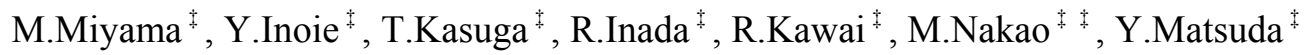 \\ Kanazawa University ${ }^{\ddagger}$, Eizo Nanao Corporation ${ }^{\ddagger}$
}

\begin{abstract}
This paper describes a $158 \mathrm{MS} / \mathrm{s}$ JPEG 2000 codec with an embedded block coder (EBC) based on a bit-plane and pass-parallel architecture. The EBC contains bit-plane coders (BPCs) corresponding to each bit-plane in a codeblock. An upper BPC transfers significance states and sign bits to a lower BPC via a first-in-first-out buffer (FIFO) to synchronize BPCs themselves. The upper and the lower bit-plane coding overlap in time with a 1-stripe and 1- column gap. The bit-modeling passes in the bitplane also overlap in time with the same gap. These methods support not only vertically causal mode, but also regular mode, which enhances the image quality. Furthermore, speculative decoding is adopted to increase throughput. This codec LSI was designed using $0.18 \mu \mathrm{m}$ process. The core area is $4.7 \times 4.7 \mathrm{~mm}^{2}$ and the frequency is $160 \mathrm{MHz}$. It is applicable to a wireless PC display.

Index Terms- JPEG 2000, EBCOT, VLSI
\end{abstract}

\section{INTRODUCTION}

JPEG 2000 is an image compression standard, featuring that high compression ratio and high-quality imaging with little block noise [1]. It is used for digital cinema encoding as well as still image encoding. Encoding with JPEG 2000 starts with discrete wavelet transform (DWT), followed by quantization and embedded block coding with optimized truncation (EBCOT). The DWT decomposes a tile image into wavelet sub-bands. The wavelet coefficients in each sub-band are scalar quantized. After quantization, a sub-band is divided into several code-blocks. The EBCOT includes bit-plane coding and context-adaptive arithmetic coding.

In the EBCOT, quantized coefficients in each codeblock are coded bit-plane by bit-plane from the most significant bit-plane to the least significant bit-plane. Bit modeling produces a pair of context and decision (CX-D) for each bit on a bit-plane. Bit modeling on a bit-plane is divisible into 3 passes: a significant propagation pass (SP), a magnitude refinement pass (MR), and a cleanup pass (CU). Each bit on the bit-plane is modeled by one of the three passes. The pass-decision for each bit depends on significance states of eight coefficients surrounding the coefficient including the bit. The significance state is defined for each quantized coefficient. A coefficient is significant on a bit-plane lower than or equal to the bitplane on which the first ' 1 ' appears when scanning the coefficient from the most significant to the least significant bit. Pairs of CX-D are provided to contextadaptive arithmetic coding. A code stream is obtained after arithmetic coding. The EBCOT is a complicated, time-consuming task. A high-performance EBCOT architecture is necessary to develop a high-throughput JPEG 2000 codec.

Several EBCOT architectures have been proposed [2][4]. One EBCOT architecture proposed in [2] includes two bit-plane coders, which encode two bit-planes in parallel. The bit-plane coder executes an SP pass, an MR pass, and a CU pass on a bit-plane also in parallel. Most bits in an upper bit-plane are usually coded with the CU pass and most bits in a lower bit-plane are usually coded with the MR pass. This architecture cannot achieve high throughput in these cases.

Word-level parallel architecture is proposed in [3]-[4]. Each bit of a quantized coefficient can be encoded in parallel because the neighbor significance states necessary for the current context calculation are known at encoding. At decoding, the neighbor states are known after the completion of the previous bit decoding. Pipeline processing stalls because the current bit decoding cannot start before completion of the previous bit decoding. This architecture supports only the vertically causal mode, whose neighbor dependency is simpler than that of regular mode.

The novel EBCOT architecture enables encoding and decoding of all bit-planes in a code-block in parallel. Three bit-modeling passes on a bit-plane coding are also executed in parallel. This architecture supports not only the vertically causal mode but also the regular mode, which enhances image quality. A JPEG 2000 codec LSI with EBCOT based on the architecture achieves $158 \mathrm{M}$ sample/s throughput. This LSI can encode and decode not only HD $(1920 \times 1080,30 \mathrm{fps}, \mathrm{YCbCr} 4: 2: 2,124 \mathrm{M}-$ sample/s), but also SXGA $(1280 \times 1024,60 \mathrm{fps}, \mathrm{YCbCr}$ 4:2:2, $158 \mathrm{M}$-sample/s) video in real-time.

\section{PARALLEL EBCOT METHODS}

Figure 1 shows a bit-plane parallel method. All bit-planes in a code-block are coded in parallel. An upper and a lower bit-plane coding overlap in time with one stripe (= four rows) and one column gap to enable reference of the upper significance states on the lower bit-plane. As shown in Fig.1, the significance state of bit ' $i$ ', which is obtainable after decoding of bit ' $i$ ' on the upper bit-plane, is necessary for bit-modeling of bit 'e' on the lower bitplane in the regular mode. The one-stripe and one-column gap is necessary to support the regular mode. The vertically causal mode does not require significance states over the boundary between stripes (' $\mathrm{g}$ ', ' $\mathrm{h}$ ', ' $\mathrm{i}$ ' for 'e'). One column gap is necessary to support the vertically causal mode. 

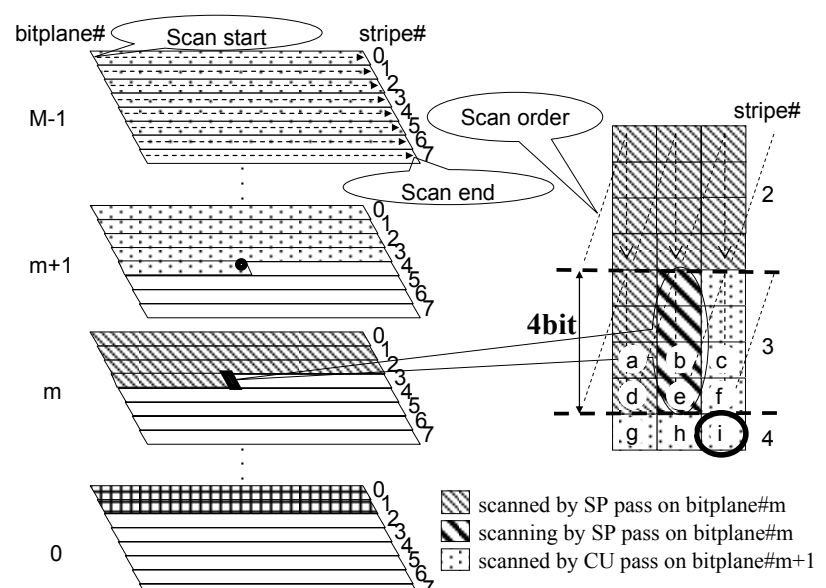

Fig. 1. Bit-plane parallel method.

Figure 2 shows a pass-parallel method. Bit-modeling passes on a bit-plane coding overlap in time with one stripe and one column gap. Figure 2 shows the passdetermination method. For the eight scanning bits shown in the figure, each pass is determined in a step. An SP pass scans the lower right four bits. An MR and a CU pass scan the upper left four bits. Then only the selected bits are modeled bit by bit. The MR and the CU pass can scan the same four bits simultaneously because the MR pass does not change the significance state.

stripe\#

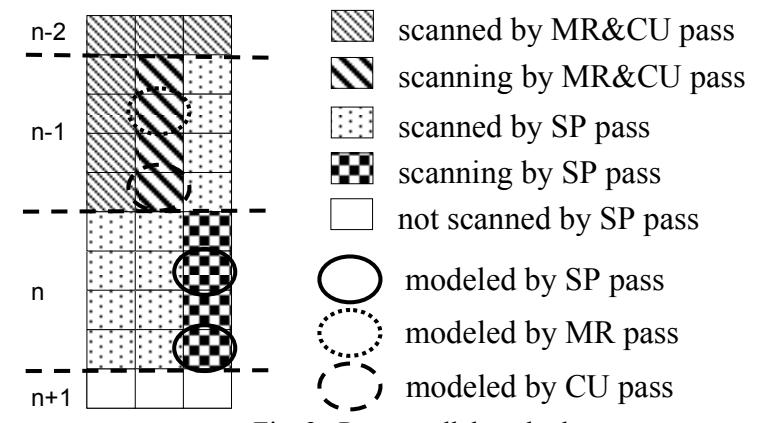

Fig. 2. Pass parallel method.

Figure 3 shows a timing diagram with these overlap methods compared to that of the conventional method. These overlap methods increase coding throughput and reduce the coding latency and data hold time of a codeblock, resulting in smaller code-block and code stream buffers.

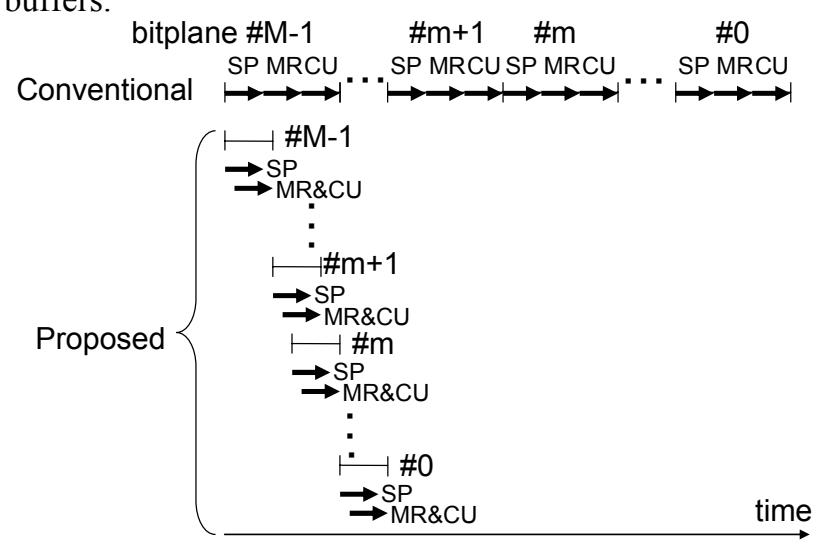

Fig. 3. Timing diagram.

Figure 4 shows one bit-modeling method for the bit possibly modeled by the SP pass at decoding. Decoding of the bit starts before complete decoding of the previous bit. Two successive bits of the same pass are modeled using another speculative method. Decoding of the lower bit starts speculatively before complete decoding of the upper bit. The method generates the lower context assuming that the upper bit is not significant. If the upper bit becomes significant after decoding, then the context is modified in an MQ decoder. These speculative methods increase the decoder throughput.

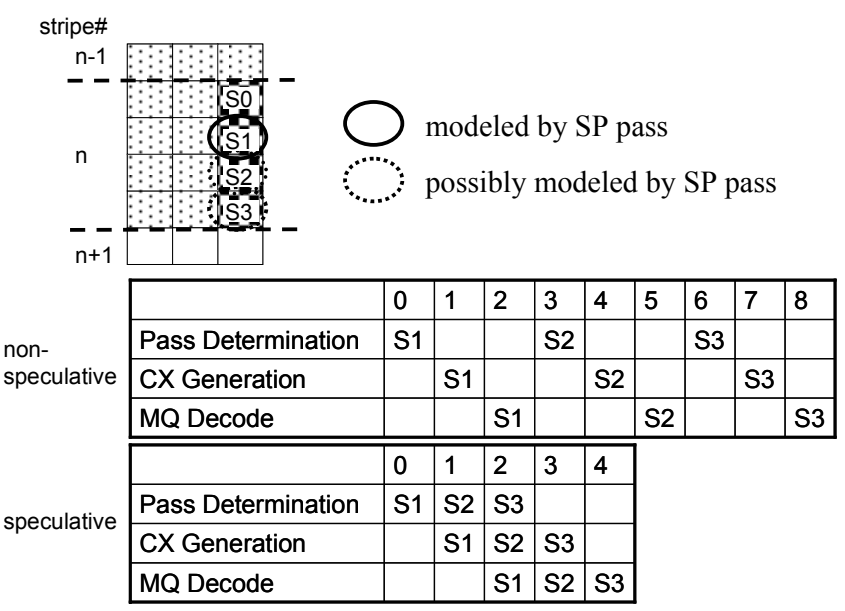

Fig. 4. Speculative method for SP pass decoding.

\section{PARALLEL EBCOT ARCHITECTURE}

\subsection{EBCOT ARCHITECTURE}

An EBCOT block diagram is shown in Fig. 5. The EBCOT consists of a code-block buffer (CBB), an embedded block coder (EBC), and a code-stream buffer (CSB). The CBB stores quantized coefficients by the code-block. Each bit-plane in a code-block is accessible in parallel. The EBC is an embedded block encoder/decoder. The EBC consists of bit-plane coders (BPCs) as the same number of bit-planes in a code-block. Each bit-plane of the CBB connects two-way to a BPC one by one. The CSB stores a code stream for each pass on a bit-plane. The CSB is divided into areas corresponding to each bitplane.

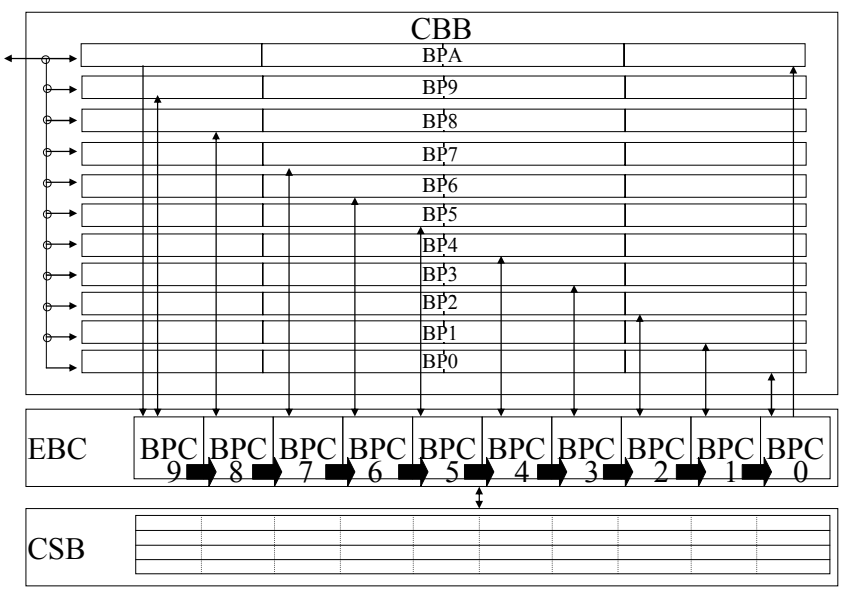

Fig. 5. EBCOT block diagram.

Each BPC encodes or decodes the corresponding bitplane at a time. At encoding, a bit-plane that stores sign 
bits (BPA) supplies sign bits to the uppermost BPC. Each bit-plane supplies quantized coefficients to the corresponding BPC. A first-in-first-out buffer (FIFO) connects an upper BPC to a lower BPC. The upper BPC sends significance states and sign bits after encoding to the lower BPC via the FIFO. At decoding, each BPC provides quantized coefficients obtained by decoding to the corresponding bit-plane. The upper BPC sends significance states and sign bits to the lower BPC. The least significant BPC provides final sign bits to the bitplane for sign (BPA). Upper bit-plane coding and lower bit-plane coding are self-synchronized by connecting BPCs with FIFO and transferring significance states and sign bits from the upper BPC to the lower BPC via FIFO.

A synchronization mechanism to access to the $\mathrm{CBB}$ or the CSB is described here. Coding of the upper bit-plane always finishes before coding of the lower bit-plane because of the significance-state dependency. After the new code-block written to a bank from the external, the most significant bit-plane in the bank can be read. After writing a result corresponding to the least significant bitplane in a bank, the code-block in the bank is legible from the external. Figure 6 shows 4-bit registers val9 and val0, which respectively corresponds to the most significant and the least significant bit-plans, to synchronize reading from and writing to the buffers. The register value varies according to the access to the most significant bit-plane, the least significant bit-plane, and a bank.

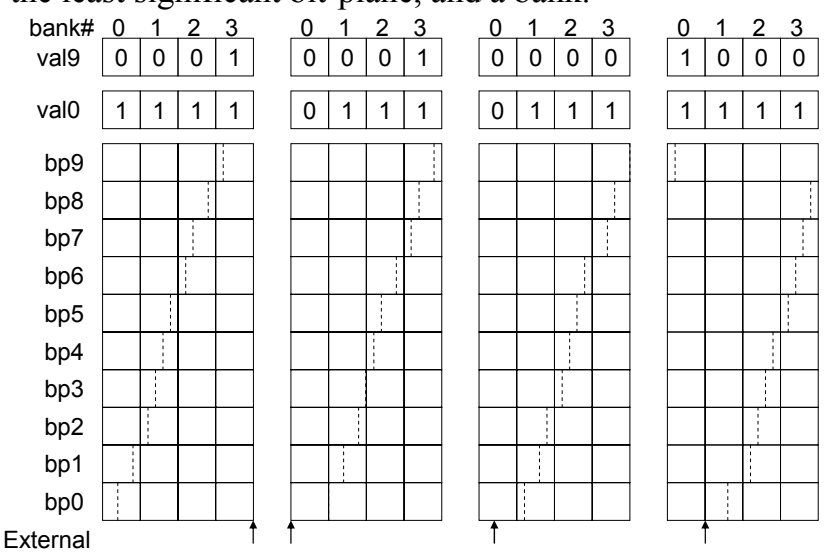

Fig. 6. Synchronization mechanism for CBB and CSB access

\subsection{BIT-PLANE CODER ARCHITECTURE}

Figure 7 shows a BPC block diagram. The BPC encodes or decodes one bit-plane at a time. The BPC consists of an SSB, a PP, a FIFO, a CXD, an MQC, a CRW, an MQD, a UPD, and an SSW. The SSB is a significance state and sign buffer. The SSB stores significance states and quantized coefficients. The PP is a pre-processor for bitmodeling. The PP determines the pass for each bit and sends the neighbor significance states, the neighbor sign bits, a quantized coefficient bit, and a kind of pass at every cycle bit-by-bit to the FIFO, which connects the PP to the CXD. The CXD is a context and decision (CX-D) generator. The CXD generates a pair of CX-D using the data from the PP at encoding. The CXD generates a context using the data from the PP and the UPD at decoding. The MQC is an MQ encoder. The MQC receives a pair of CX-D generated by the CXD, and produces a code stream using context-adaptive arithmetic coding. The CRW writes a code stream to the CSB at encoding; it reads the code stream from the CSB at decoding. The MQD is an MQ decoder, which produces a decision using the code stream from the CSB, the context, and the kind of pass from the CXD. The UPD updates the past significance states and sign bits of the current bit using decisions decoded by the MQD. Future significance states and sign bits of the current bit are provided from the PP. The SSW writes the significance states, sign bits, and quantized coefficients to the SSB provided by the PP at encoding. At decoding, the significance states and sign bits provided by the MQD overwrite old ones provided by the PP. The SSW writes the overwritten data to the SSB.

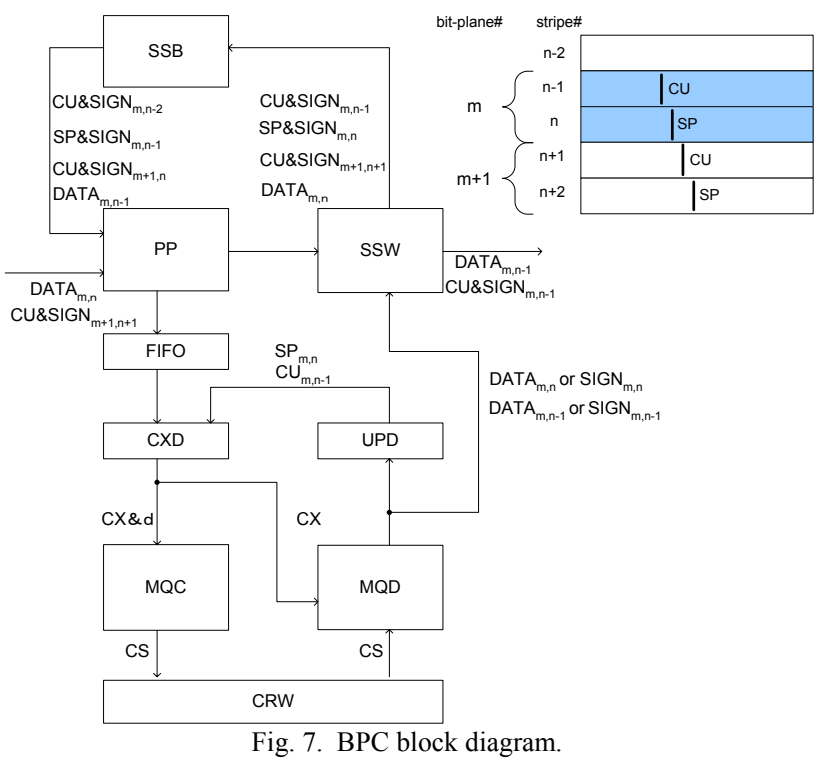

At decoding, the PP speculatively produces the neighbor significance states and sign bits around the bit that is possibly modeled by an SP pass to the FIFO before complete decoding of the previous bit. The possibly modeled bit is located as a neighbor of the bit modeled by the SP pass. No neighbor state around the bit is significant. Its own state is not significant. The MQD decodes the bit if the upper state becomes significant after decoding; it does not decode the bit if the upper state does not become significant after decoding. In the situation, the MQD informs the SSW that the bit is not modeled by the SP pass. The bit can be modeled by a later CU pass.

When two successive bits of the same pass are decoded, the CXD generates the lower context assuming the upper bit is not significant before complete decoding of the upper bit. The CXD informs the MQD that the lower bit is modeled speculatively. The MQD modifies the lower context if the upper bit becomes significant. This method simplifies the MQD logic comparing to that of the method generating the true context again from the neighbor significance states. Re-calculation of the context and the MQ decoding can be included in the same pipeline stage; thereby, the decode pipeline dose not stall. Speculative decoding achieves throughput of one sample per cycle.

The SSB stores significance states, sign bits, and quantized coefficients for three stripes, not for all stripes, 
on a bit-plane. The significance state is represented by two bits instead of the three bits of the conventional case. With our method, state 0 represents that the bit is not significance. State 1 represents that the bit is significant and has not been scanned by the MR pass in the same bitplane on which the bit becomes significant in the SP pass. State 2 represents that the bit is significant and has not been modeled by the first MR pass. State 3 represents that the bit is significant and has been modeled by the first MR pass. This method reduces the SSB capacity.

\section{JPEG 2000 CODEC LSI}

Figure 8 shows a JPEG 2000 codec block diagram. The circuit contains a VIOIF, an ITRAN, an EBCOT, and a CPUIF. The ITRAN contains a CCNV, an LB, a DWT, a $\mathrm{WB}$, and a $\mathrm{Q}$. The $\mathrm{CCNV}$ is an $\mathrm{RGB}-\mathrm{YCbCr}$ color converter. The DWT executes DWT or inverse DWT. The $\mathrm{Q}$ executes quantization or inverse quantization. These circuits are reconfigurable and can operate in both directions, resulting in gate reduction. The EBCOT executes embedded block coding and distortion calculation. In addition to an ordinary rate-distortion optimization, an external controller progressively enhances quality on whole image across tile boundaries within a limited bandwidth [5]. The JPEG 2000 codec achieves throughput of one sample per clock cycle.

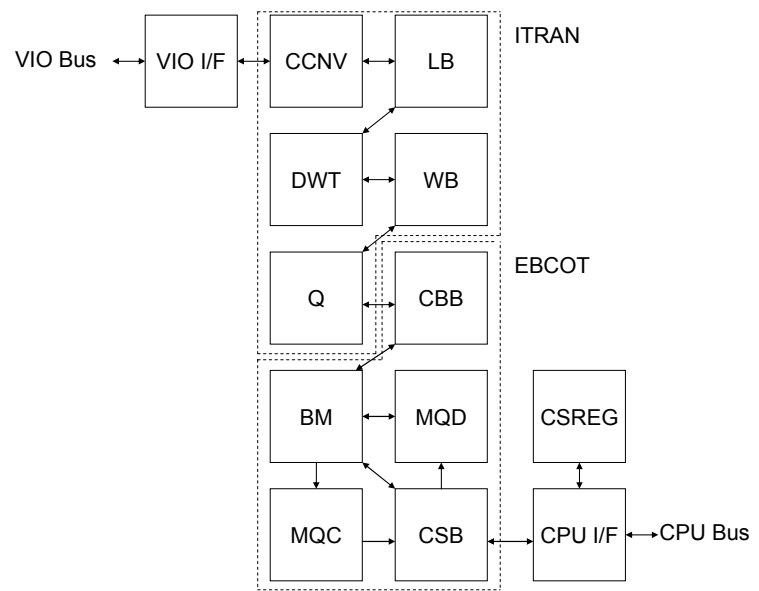

Fig. 8. JPEG 2000 codec block diagram.

The JPEG 2000 codec LSI was designed using $0.18 \mu \mathrm{m}$ process. Table 1 shows the LSI specification. Figure 9 shows a plot image of the codec LSI. The estimated operating frequency and power consumption of the codec are $160 \mathrm{MHz}$ and $1.5 \mathrm{~W}$, respectively.

Table 1. JPEG 2000 codec specification.

\begin{tabular}{|l|l|}
\hline Function & $\begin{array}{l}\text { JPEG2000 codec, regular } \\
\text { mode supported }\end{array}$ \\
\hline Performance & $158 \mathrm{M}-$ Sample/s \\
\hline Tile size & $128 \times 128$ pixels \\
\hline Codeblock size & $32 \times 32$ pixels \\
\hline Gate count & $1,084 \mathrm{k}$ \\
\hline Area (Core) & $4.7 \times 4.7 \mathrm{~mm}^{2}$ \\
\hline Frequency & $160 \mathrm{MHz}$ \\
\hline Process & $0.18 \mathrm{um}, 6$ metal layers \\
\hline
\end{tabular}

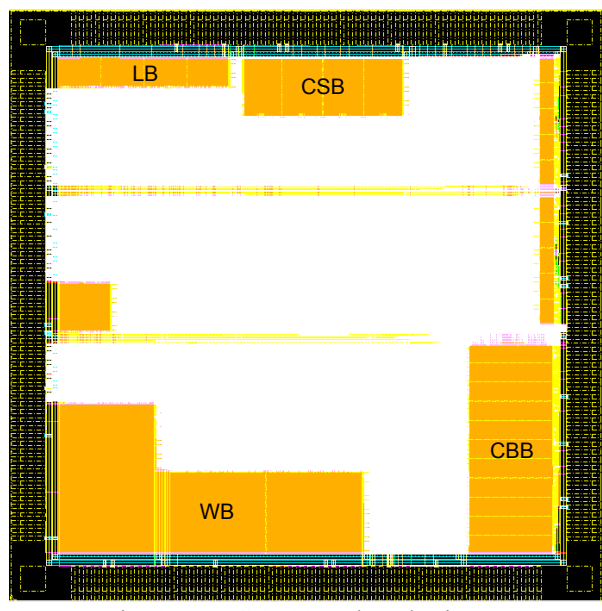

Fig. 9. JPEG 2000 codec plot image.

\section{CONCLUSION}

This paper presented a JPEG 2000 codec LSI with an embedded block coder based on a bit-plane and passparallel architecture. An upper bit-plane and a lower bitplane coding overlap in time with 1-stripe and 1-column gap. Bit-modeling passes also overlap in time with the same gap. These methods support not only the vertically causal mode but also the regular mode to enhance image quality, while providing short latency and high throughput of embedded block coding. Furthermore, speculative decoding is used to increase the throughput. The codec was implemented with $0.18 \mu \mathrm{m}$ process. The core area is $4.7 \times 4.7 \mathrm{~mm}^{2}$ and the estimated frequency is $160 \mathrm{MHz}$. The codec achieves $158 \mathrm{MS} / \mathrm{s}$ throughput and is applicable to wireless PC display with both high performance and high image quality [5].

\section{ACKNOWLEDGMENTS}

This work is supported by VLSI Design and Education Center (VDEC), the University of Tokyo in collaboration with Cadence Design Systems, Inc. and Synopsys, Inc.

\section{REFERENCES}

[1] "Information Technology; JPEG 2000 Image Coding System- Part 1: Core Coding System”, ISO/IEC 15444-1, Aug.2000.

[2] Hideki YAMAUCHI, Shigeyuki OKADA, Kazuhiko TAKETA, Tatsushi OHYAMA, "A Single-Chip JPEG 2000 Encode Processor Capable of Compressing D1-Images at 30 frame/s without Tile Division", IEICE TRANS. ELECTRON., VOL.E87-C, NO.4 APRIL 2004.

[3] Hung-Chi Fang, Chao-Tsung Huang, Yu-Wei Chang, TsuChih Wang, Po-Chih Tseng, Chung-Jr Lian, Liang-Gee Chen, "81 MS/s JPEG 2000 Single-Chip Encoder with RateDistortion Optimization”, ISSCC2004 18.2.

[4] Hung-Chi Fang, Yu-Wei Chang, Tu-Chih Wang, Chung-Jr Lian, and Liang-Gee Chen, "Parallel Embedded Block Coding Architecture for JPEG 2000", IEEE TRANSACTIONS ON CIRCUITS AND SYSTEMS FOR VIDEO TECHNOLOGY, VOL.15, NO.9, SEPTEMBER 2005.

[5] M. Nakao, et al, "Low delay and Real-time Image Transmission Hardware for Remote Desktop", SID2007, Jun. 2007. 